\title{
EFFECTS OF DIRECT AND INDIRECT SOLAR RADIATION PRESSURE IN ORBITAL PARAMETERS OF GPS SATELITTES
}

\author{
Sergiu Lupu and Eugen Zaharescu
}

\begin{abstract}
In this paper we determined the variation of the Keplerian orbital elements of a GPS satellite due to the direct and indirect action of solar radiation pressure. For this study, we created a soft program to determine the Keplerian elements. This soft uses the initial conditions of position and speed of a GPS satellite and solves the Laplace integration problem using Runge - Kutta algorithm of $4^{\text {th }}$ order.
\end{abstract}

\section{Introduction}

The vectorial differential equation of the relative motion of a satellite around the central body, in the absence of any disturbing influences, has the following form [1-2]:

$$
\frac{d^{2} \vec{r}}{d t^{2}}=-\mu \frac{\vec{r}}{r^{3}}(\mathbf{1})
$$

where:

$\mu=G M-$ Earth's gravitational constant,

$\vec{r}$ - the vector from the center of mass of the central body to the satellite.

Key Words: artificial satellites, Laplace problem, solar radiation pressure, orbital elements, Runge - Kutta algorithm.

2010 Mathematics Subject Classification: Primary 70F15, 65L06.

Received: June 2013

Revised: September 2013

Accepted: November 2013 
On an artificial satellite of the Earth, act a significant number of disturbing forces (accelerations). These are much smaller (as magnitude) than the central force, but that significantly influences the dynamics, in time.

Depending on their nature, there are gravitational and non-gravitational perturbation accelerations.

There are two methods can be used in order to calculate the orbits of the GPS satellite.

The first one is based on the analytical solutions of the Lagrange planetary equations, expressed in Keplerian orbital elements terms.

The second method is based on the numerical solution of the second order differential equation of the disruptive relative movement:

$$
\frac{d^{2} \vec{r}}{d t^{2}}=-\mu \frac{\vec{r}}{r^{3}}+\frac{\partial \vec{R}}{\partial r} \cdot(\mathbf{2})
$$

where:

$\frac{\partial \vec{R}}{\partial r}$ - represents the sum of all perturbing accelerations.

For GPS satellites, the module of the central acceleration $\left(-\mu \frac{\vec{r}}{r^{3}}\right)$ is $10^{4}$ times bigger than the sum of all these disruptive accelerations.

The numerical solution of the GPS satellites orbits is based on the direct numerical integration of the second order differential equations of the disrupted relative movement.

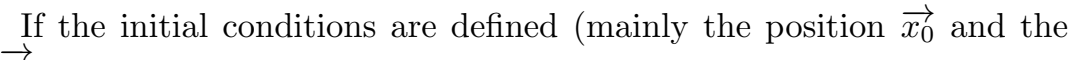
speed $\dot{x}_{0}$ considered at launching time), the equations can be numerically integrated [3].

The Keplerian orbit will be cross-referenced as well. Thus, only the small differences between the total acceleration and the central acceleration will have to be integrated. As a result, the precise position will be given by the sum of the (incremental) growth and the position vector on the referenced ellipse.

The system of 2 first order differential equations is:

$$
\begin{gathered}
\overrightarrow{\dot{x}(t)}=\overrightarrow{\dot{x}\left(t_{0}\right)}+\int_{t_{0}}^{t}\left[\overrightarrow{\overrightarrow{d x}\left(t_{0}\right)}-\frac{\mu}{r^{3}\left(t_{0}\right)} \overrightarrow{x\left(t_{0}\right)}\right] d t .(\mathbf{3}) \\
\overrightarrow{x(t)}=\overrightarrow{x\left(t_{0}\right)}+\int_{t_{0}}^{t} \overrightarrow{\dot{x}\left(t_{0}\right)} d t
\end{gathered}
$$

The numerical integration of this system is made by applying a fourth order Runge Kutta algorithm [4]. 
The perturbing acceleration magnitude depends on the type of the phenomenon that occurs, and on the other hand, on the values of satellite orbital parameters.

The effects of a third-body travelling in a circular orbit around a main body on a massless satellite that is orbiting the same main body have been studied in several papers [5].

Of all non-gravitational perturbations, the most important is the solar radiation pressure. Its mode of action on an artificial satellite of the Earth is direct and indirect [6].

The soft program has determined the values of the range and velocity vectors for each integration step, for the selected period range, which it was written in a text file [7].

The program also solved the Laplace problem [8] and determined the all known Keplerian elements $(a, e, i, \omega, \Omega, M)$.

\section{Direct solar radiation pressure effects}

Considering the disturbing acceleration formula:

$$
F_{\text {direct }}=k^{\prime} \frac{A}{M} q \Psi \frac{\overrightarrow{r_{\text {sat }}}-\overrightarrow{r_{\text {Sun }}}}{\overrightarrow{r_{\text {Sun }}}} \text {.(4) }
$$

the variations of the Keplerian elements were determined (Fig. 1).

where:

$k \prime$ - constant of the reflectivity of the satellite.

$A$ - the area of transverse section of the satellite, perpendicular to the disturbing force.

$M$ - the satellite mass.

$q$ - the ratio of the solar constant and the speed of light.

$\Psi$ - the shadow function which has value 1 if the satellite is fully illuminated and 0 if it is in the Earth's shadow.

$\overrightarrow{r_{s a t}}$ - the geocentric vector of the satellite

$\overrightarrow{r_{S u n}}$ - the geocentric vector of the Sun

Thus, for calculations we will consider $k \prime=1$ if the reflection or absorption of sunlight by the exposed area of the satellite is total and $k^{\prime}=1.44$ if the reflection is diffuse.

The semi-major axis has a short-periodic perturbation with a period of 6 hours superimposed over a secular perturbation. For an orbital period, the semi-major axis suffers a 4 meters variation. 
EFFECTS OF DIRECT AND INDIRECT SOLAR RADIATION PRESSURE IN

1.jpg
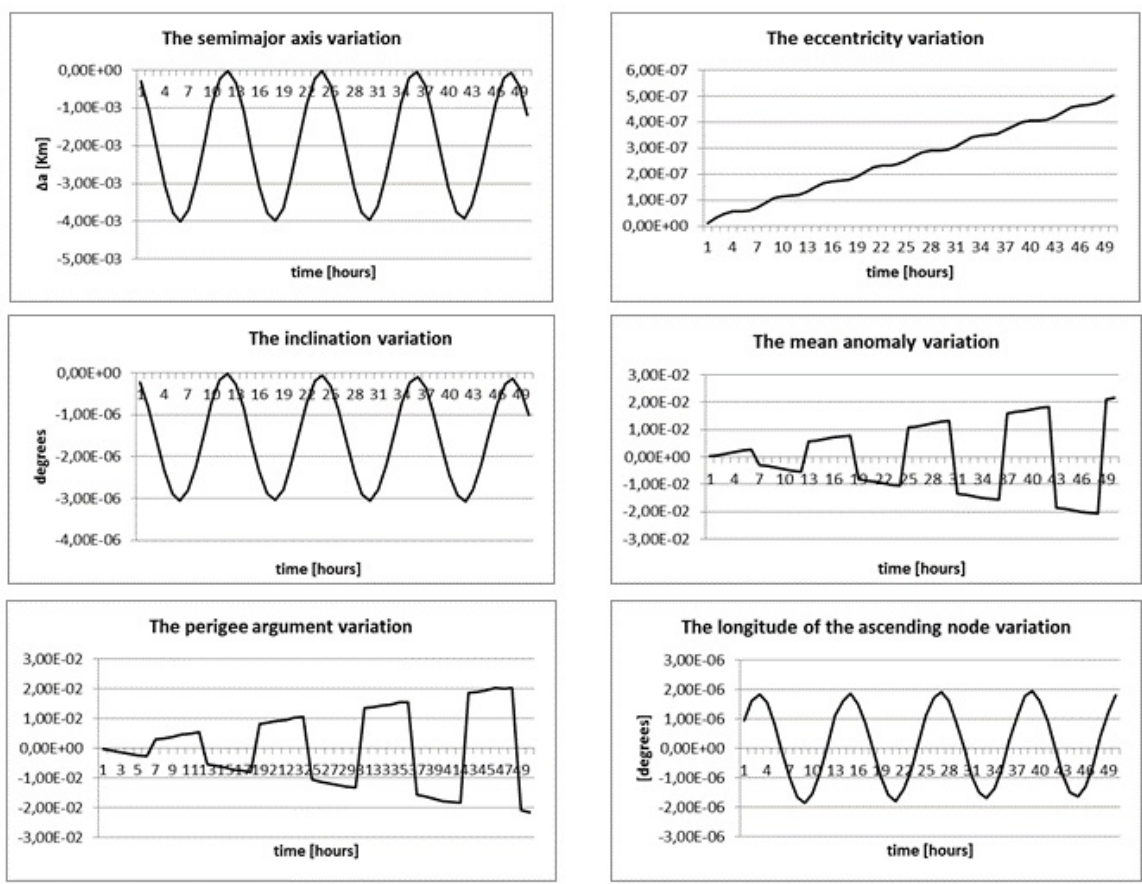

Figure 1: The variation of the Keplerian elements due to the direct solar radiation action

The eccentricity has a secular perturbation superimposed on a shortperiodic perturbation with a period of 6 hours and the order of magnitude $6 \cdot 10^{-8}$ degrees.

The inclination has the same type of perturbation as the semi-major axis, a short-periodic perturbation superimposed on a secular perturbation. Short-period perturbation has the period of 6 hours and the order of magnitude $3 \cdot 10^{-6}$ degrees.

The longitude of the ascending node suffers a short periodic perturbation with a period of 6 hours and amplitude of $1,7 \cdot 10^{-6}$ degrees, superimposed over a secular perturbation. 


\section{$3 \quad$ Indirect solar radiation pressure effects}

\subsection{Earth's radiation model}

The Earth's atmosphere reflects some of the incoming radiation from the Sun, and for simplicity we will consider that this radiation is reflected back into space by a sphere covered with a Lambertian surface [9 - 11].

The incoming radiation from the Sun which intersects with the Earth can be determined as $A_{E} E_{S u n}$ where $A_{E}$ represent Earth's disc area and $E_{S u n}$ is the solar constant. A fraction of this radiation in the visible range is immediately reflected, another part is absorbed and another one is re-emitted as infrared radiation [12].

The irradiance vector for a satellite found at $h$ altitudes, with $\vec{r}$ direction vector, due to Earth's overall albedo, depends only on the relative positions of the satellite, Earth and Sun ( $\psi$ angle) (Fig. 2).

The Earth's radiation model is the sum of the reflected and emitted radiation and it can be expressed as:

$$
E_{\text {sat }}(\psi)=\frac{A_{E} E_{\text {Sun }}}{\left(R_{E}+h\right)^{2}}\left[\frac{2 \alpha}{3 \pi^{2}}((\pi-\psi) \cos \psi+\sin \psi)+\frac{(1-\alpha)}{4 \pi}\right] .(5)
$$

where:

$A_{E}=\pi R_{E}$

$R_{E}=6371 K m-$ Earth's medium radius

$h=2655 \mathrm{Km}-$ GPS satellites altitude

$E_{\text {Sun }}=1367 \mathrm{~W} / \mathrm{m}^{2}[13]$

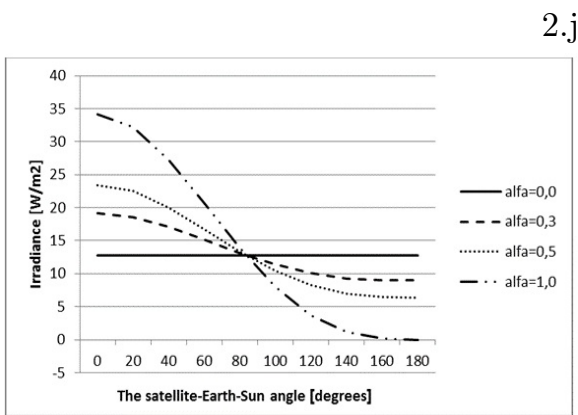

a)

2.jpg

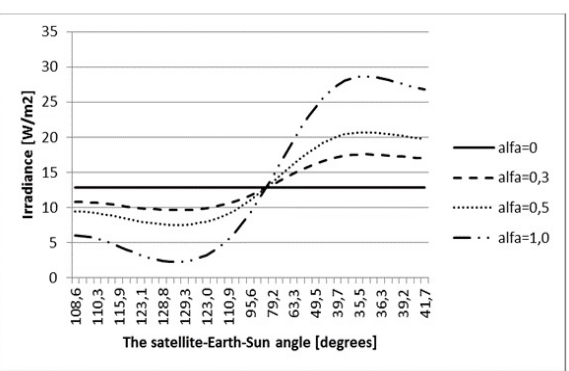

Figure 2: The Earth's total irradiance 
In Fig. 2 a) $\psi$ is the angle from 0 to 180 degrees and in Fig. 2 b) $\psi$ is the angle of the P07 GPS satellite determined by the position information of the precise ephemeris in SP3 format, available to users by JPL agency and Sun positions [14], determined by numerical integration

$$
\cos \psi=\frac{\overrightarrow{r_{\text {sat }}} \cdot \overrightarrow{r_{\text {Sun }}}}{\left|\overrightarrow{r_{\text {sat }}}\right|\left|\overrightarrow{r_{\text {Sun }}}\right|} \cdot \text { (6) }
$$

Thus, it is noted that the Earth's irradiance variation graph is a sinusoidal curve and it decreases with the increasing amount of the Earth's albedo and the increasing angle between the satellite, Earth and Sun. The irradiance is independent of the $\psi$ angle and is constantly taking different values depending on the value of the Earth's albedo. For zero value of the Earth's albedo the irradiance is also zero.

The GPS satellite on which the radiation pressure exerted by the Earth's actions will be considered as having two forms: in a first approximation as a sphere or "ball" and as a "box-wings". Of particular interest is the cross section of the satellite and its optical properties.

For the case where the satellite is approximated as a "ball" we make a simple model of the satellite by mediating the acceleration value for the $\psi$ angle between the satellite - Earth and Sun.

Thus, the acceleration can be calculated with the following formula [15]:

$$
\overrightarrow{F_{\text {indirect }}}=\frac{A}{M} \frac{E_{\text {Sun }}}{c} C_{\text {ball }} \frac{\overrightarrow{r_{\text {sat }}}}{r_{\text {sat }}}(\mathbf{7})
$$

where $c$ is speed of light and $C_{b a l l}$ is a numerically determined constant which gives the average size and optical properties of GPS satellites [16], [17] (Table 1).

Table 1. GPS SATELLITES PARAMETERS (SATELLITES BALL TYPE)

\begin{tabular}{|c|c|c|}
\hline Type of GPS satellite & $\frac{\text { Area/Mass ratio }}{\left[\mathrm{m}^{2} / \mathrm{Kg}\right]}$ & $C_{\text {ball }}$ \\
\hline Block I & 0.01513 & 0.8876 \\
\hline Block II & 0.01667 & 0.8551 \\
\hline Block IIR & 0.01606 & 0.8134 \\
\hline
\end{tabular}

In the next figure (Fig. 3), the graphs of variation of Keplerian elements due to indirect solar radiation pressure action can be observed.

The semi-major axis has a short-periodic perturbation equal to the orbital period (12 hours) and a variation of 4 centimeters.

The eccentricity shows a short-periodic perturbation equal to the orbital period (12 hours) and with the order of magnitude $2,07 \cdot 10^{-8}$. 
The variation of orbital inclination made in 50 hours is zero. From the graph of variation made in 500 hours it can be observed that the orbital inclination has a secular perturbation with a variation of $1 \cdot 10^{-8}$ degrees.

The mean anomaly has mixed periodic perturbations:

- a short periodic perturbation equal to the orbital period with an amplitude of $1 \cdot 10^{-3}$ degrees;

- a short periodic perturbation equal to the orbital period with an amplitude of $2 \cdot 10^{-3}$ degrees;

- a secular perturbation.

3.jpg
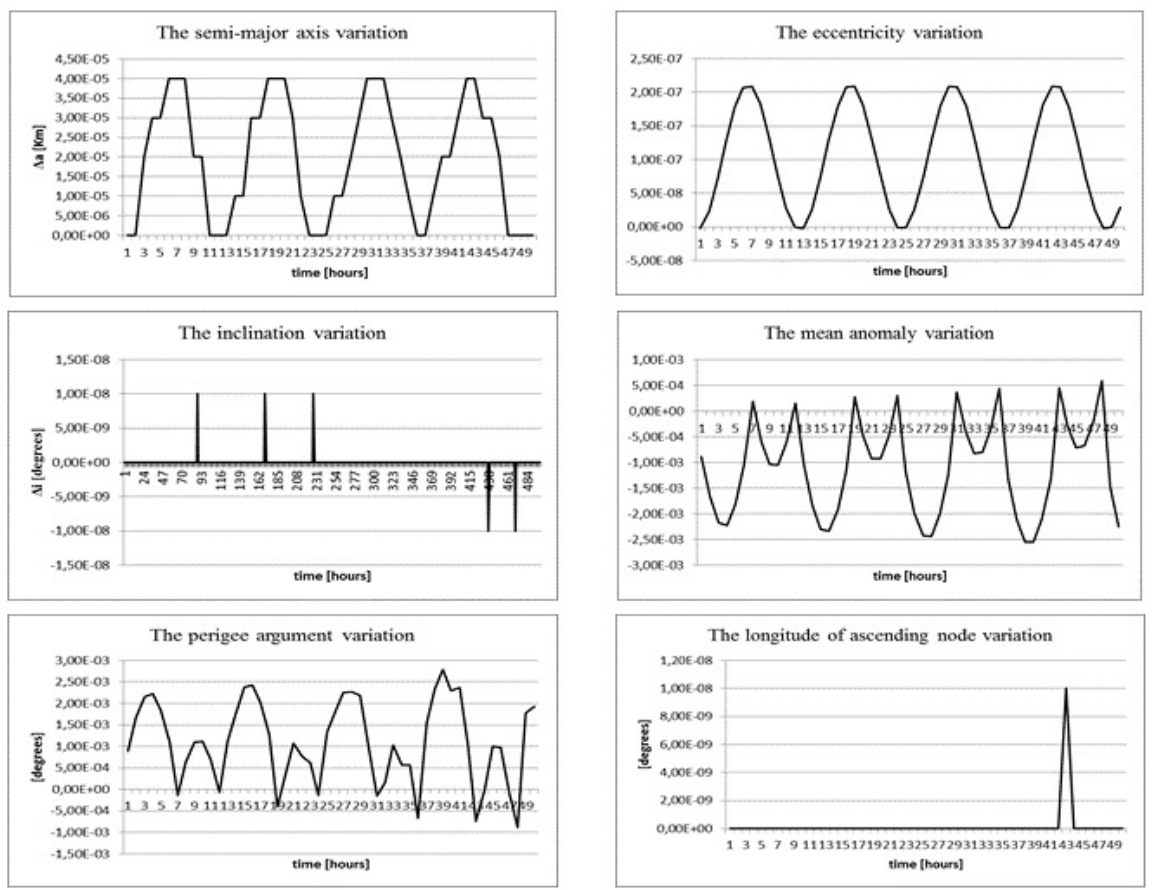

Figure 3: The variation of the Keplerian elements due to the direct solar radiation action

The perigee argument has mixed periodic perturbations:

- a short periodic perturbation with 6 hours period with an amplitude of $1 \cdot 10^{-3}$ degrees 
- a short periodic perturbation equal to the orbital period with an amplitude of $2,5 \cdot 10^{-3}$ degrees;

- a secular perturbation.

The longitude of the ascending node shows just a secular perturbation with the order of magnitude $1 \cdot 10^{-8}$ degrees.

\section{Conclusions}

The main goal of this paper was to present the variation of all the Keplerian elements due to direct and indirect solar radiation pressure action.

As a result, by the direct solar radiation pressure action, all the Keplerian elements suffer short periodic perturbations with a 6 hours period and secular perturbations.

The indirect effect of the solar radiation pressure introduces short periodic perturbations with the period equal to the orbital period superimposed over secular perturbations.

In this case, the orbital inclination variation shows just a secular perturbation, because the inclinations of the GPS satellites are very stable.

This information about the variation types and values of the Keplerian orbital elements of a GPS satellite, due to the direct and indirect action of solar radiation pressure, can improve the precise location of the GPS satellites in space in real time.

This data can be utilized at the master station situated in Colorado, U.S.A. in order to compute and upload the precise locations of the GPS satellites in space. .

\section{References}

[1] D. Brouwer, G.M. Clemence. (1961). Methods of Celestial Mechanics, Academic Press, New York.

[2] Vallado, D. A. (1997). Fundamentals of Astrodynamics and Applications", McGraw-Hill, New York.

[3] B. Hofmann-Wellenhof, et al. (1994). Global Positioning System - Theory and Practice, New York.

[4] G. Beutler. (2005). Methods of Celestial Mechanics", I \& II. Springer, Germany.

[5] Carlos Renato Huaura Solórzano and Antonio Fernando Bertachini de Almeida Prado. (2013). A Comparison of Averaged and Full Models to Study the Third-Body Perturbation. The Scientific World Journal. 
[6] A. Milani, A.M. Nobili, P. Farinella. (1987). Non-graviational Perturbations and Satellite Geodesy. Adam Hilger.

[7] S. Lupu. (2011). Elemente de dinamica sistemului global de pozitionare NAVSTAR - GPS. Academia Navala Mircea cel Batran, Constanta.

[8] G. Seeber. (1993). Satellite Geodesy, Foundation, methods and applications. Walter de Gruyter, Berlin.

[9] N. Borderies, PY. Longaretti. (1999). A new treatment of the albedo radiation pressure in the case of a uniform albedo and of a spherical satellite. Celestial Mechanics and Dynamical Astronomy.

[10] A. J Chapman. (1984). Heat Transfer. Macmillan.

[11] J. Qiu, PR Goode, E. Pallé, V. Yurchyshyn, J. Hickey, P. Montañes Rodriguez, MC. Chu, E. Kolbe, CT. Brown, SE. Koonin. (2003). Earthshine and Earth's albedo: 1. Earthshine observations and measurements of the lunar phase function for accurate measurements of the Earth's Bond albedo. Journal of Geophysical Research.

[12] F W. Taylor. (2005). Elementary Climate Physics. Oxford University Press, United Kingdom.

[13] C. Frolich, J. Lean. (1998). The Sun's Total Irradiance: Cycles, Trends and Related Climate Change Uncertainties since 1976. Geophysical Research Letters, vol. 25, no. 23, pp. 4377-4380.

[14] http://www.iers.org

[15] Rodriguez-Solano, C. J.; Hugentobler, U.; Steigenberger, P.; Lutz, S. (2012). Impact of Earth radiation pressure on GPS position estimates. Journal of Geodesy, Springer, vol. 86, no. 5, pp. 309-31\%.

[16] H. Fliegel, T. Gallini, E. Swift. (1992). Global Positioning System Radiation Force Model for Geodetic Applications. Journal of Geophysical Research, pp. 559-568.

[17] H. Fliegel, T. Gallini. (1996). Solar Force Modelling of Block IIR Global Positioning System satellites. Journal of Spacecraft and Rockets, pp. 863866.

Sergiu LUPU,

Department of Navigation and Naval Management,

"Mircea cel Batran" Naval Academy,

1, Fulgerului Street, Constanta, Romania

e-mail: sergiu.lupu@anmb.ro 
EFFECTS OF DIRECT AND INDIRECT SOLAR RADIATION PRESSURE IN ORBITAL PARAMETERS OF GPS SATELITTES

Eugen ZAHARESCU,

Department of Mathematics and Computer Science,

OVIDIUS University of Constanta,

124, Mamaia Boulevard, Constanta, Romania

e-mail: ezaharescu@univ-ovidius.ro 